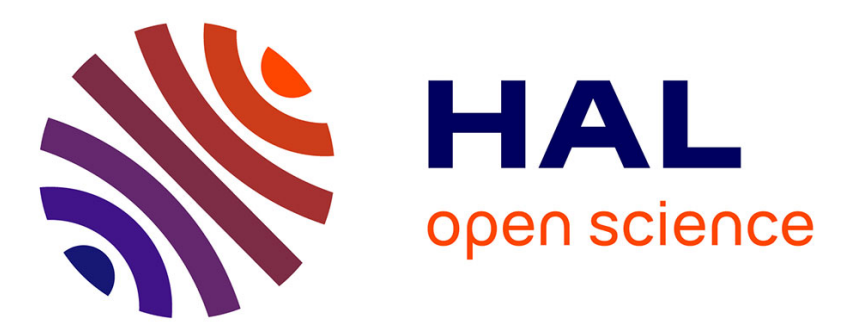

\title{
Recherche en éducation et évolution du système éducatif belge francophone
}

Marc Demeuse, Sabine Soetewey

\section{To cite this version:}

Marc Demeuse, Sabine Soetewey. Recherche en éducation et évolution du système éducatif belge francophone. Jean-François Marcel et Hervé Savy. Evaluons, évoluons. L'enseignement agricole en action, Educagri éditions, pp.173-183, 2013, AGORA, 978-2-84444-922-1. hal-00837558

\section{HAL Id: hal-00837558 https://hal.science/hal-00837558}

Submitted on 22 Jun 2013

HAL is a multi-disciplinary open access archive for the deposit and dissemination of scientific research documents, whether they are published or not. The documents may come from teaching and research institutions in France or abroad, or from public or private research centers.
L'archive ouverte pluridisciplinaire HAL, est destinée au dépôt et à la diffusion de documents scientifiques de niveau recherche, publiés ou non, émanant des établissements d'enseignement et de recherche français ou étrangers, des laboratoires publics ou privés. 


\title{
Recherche en éducation et évolution du système éducatif belge francophone
}

\author{
Marc Demeuse \\ Professeur, Institut d'Administration Scolaire, Université de Mons, Belgique \\ Sabine Soetewey \\ Chercheuse, Institut d'Administration Scolaire, Université de Mons, Belgique
}

\section{Résumé pour l'introduction}

L'évaluation n'est pas une fin en soi, mais elle est constitue une part importante de tout processus régulé. Dans le domaine de l'éducation formelle, l'évaluation prend des formes multiples et est au cœur même de l'Ecole, qu'il s'agisse de l'évaluation des élèves ou des enseignements.

Dans leur chapitre, Demeuse et Soetewey traitent principalement de l'évaluation des systèmes éducatifs et des évolutions que ce domaine a pu connaître en Belgique francophone. Ils montrent notamment comment les chercheurs ont pu prendre part aux développements de ce système éducatif et à son pilotage. A travers les évaluations internationales d'abord, principalement menées dans un but d'amélioration de la connaissance du système par les chercheurs eux-mêmes, ceux-ci ont été de plus en plus impliqués dans la mise en œuvre de nouveaux programmes et dans l'élaboration d'outils de pilotage. Les deux auteurs belges illustrent cette évolution par la présentation d'une recherche-action dans laquelle chercheurs et responsables du système éducatif co-élaborent de nouveaux outils de pilotage, mais aussi développent une meilleure compréhension du fonctionnement de l'Ecole. Plus que le développement d'outils, c'est l'évolution du système lui-même qui est visée, à travers une meilleure régulation.

A côté de cette recherche, les auteurs présentent également une étude du curriculum scientifique dans l'enseignement secondaire supérieur. Cette présentation permet de constater comment un problème de départ - améliorer la lisibilité des programmes de sciences - doit pouvoir être repensé, compte tenu de l'organisation complexe du système éducatif belge francophone et des problèmes que cette organisation génère en termes d'équité..

\section{Notice bibliographique}

Psychologue et statisticien, Marc Demeuse est professeur à l'Université de Mons (Belgique), en Faculté de psychologie et des sciences de l'éducation. Il y dirige l'Institut d'administration scolaire et est impliqué dans plusieurs projets et réseaux de chercheurs au niveau européen. Il a participé, comme expert, à la production des premiers indicateurs européens de la qualité de l'éducation scolaire (2000) et a piloté la réalisation d'un ensemble d'indicateurs d'équité des systèmes éducatifs en Europe. Il consacre une partie de ses recherches aux politiques d'éducation prioritaire ciblées (ZEP, discriminations positives...), notamment dans une perspective comparative. Il participe, comme expert, à différentes commissions et organes de pilotage du système éducatif belge francophone (Commission de pilotage, Institut de formation en cours de carrière des enseignants).

Professeur de sciences dans l'enseignement secondaire supérieur, Sabine Soetewey a intégré l'Institut d'Administration scolaire de l'Université de Mons en janvier 2010. Détentrice d'un deuxième cycle universitaire en sciences biologiques (1996) et d'un DEA en biologie des populations (1999), elle est agrégée en sciences. Elle travaille actuellement sur deux recherches concernant le réseau d'enseignement organisé par la Communauté française de Belgique. Dans ce cadre, elle s'intéresse d'une part à l'analyse 
du curriculum en sciences dans les deuxième et troisième degrés de l'enseignement secondaire ordinaire. D'autre part, elle s'intéresse au pilotage de l'enseignement au travers du développement d'outils informatisés permettant la création d'indicateurs spécifiques et la modélisation/simulation du système éducatif.

\section{Introduction}

\section{Un système éducatif historiquement très complexe et peu régulé}

Le système éducatif belge francophone, comme ses deux homologues, flamand et germanophone, est construit sur le principe constitutionnel de liberté d'enseignement. Celle-ci se décline de deux manières complémentaires: liberté de choix d'établissement scolaire pour les parents, y compris au sein de l'enseignement public, et liberté d'offre d'enseignement par des opérateurs publics et privés, ces derniers appartenant principalement au monde confessionnel, majoritairement catholique. Dans ce cadre, les écoles organisées par des opérateurs publics et privés sont subventionnées sur une base identique, en fonction principalement du nombre d'élèves accueillis.

Historiquement, c'est une opposition au régime hollandais très centraliste auquel est soumis le territoire de l'actuelle Belgique, à l'issue de la débâcle napoléonienne, qui justifie, lors de son indépendance en 1830, cette volonté très forte du Parti catholique de permettre l'installation d'un système d'enseignement privé. Ce parti imagine alors qu'il pourrait n'exister que ce type d'enseignement et que celui-ci serait même appelé un jour à être financé par l'Etat (Demeuse, 2010). Durant tout le dix-neuvième siècle et la première moitié du vingtième, tenants de l'enseignement privé catholique et défenseurs de l'enseignement public vont s'affronter, parfois fort durement, pour aboutir, en 1959, à une loi toujours d'application et qui établit un financement de toutes les écoles, publiques et privées, pour autant qu'elles se soumettent à un contrôle minimum de l'Etat. Ce contrôle ne va pas aussi loin que dans d'autres pays, notamment en France, dans le cas des écoles sous contrat, et les écoles privées disposent, par exemple, de leurs propres programmes et ne sont donc pas tenues de suivre celui de l'Etat, même si leurs programmes doivent être agréés par l'autorité publique.

Progressivement, on observe une transformation du système éducatif, construit initialement, comme le reste de la société belge, autour de "piliers », en un système qualifié de quasi-marché (Vandenberghe, 1998). Dans ce cadre, ce ne sont plus les convictions philosophiques qui guident le choix des parents vers une école publique ou une école confessionnelle, mais d'autres mobiles, dont notamment la réputation des écoles. Ces choix se marquent également au sein d'un même réseau, public ou privé, entre les écoles organisées par celui-ci dans une zone donnée. Alors même que la concurrence s'exacerbe, au moins chez les parents des élèves les plus favorisés, aucun examen ou publication ne permet d'étayer ces choix. Chaque établissement délivre ses diplômes, reconnus par tous, sur la base de ses propres programmes et évaluations, et le niveau d'enseignement suivant accueille les élèves ou les étudiants en fonction de ces " sésames locaux », sans évaluation à l'entrée (en dehors des études en sciences appliquées à l'université). L'administration de l'enseignement a, quant à elle, pour mission principale de liquider les subventions à l'enseignement privé et public subventionné (les enseignants de l'enseignement subventionné étant directement payés par cette administration, mais engagés par la direction de leur école) et d'organiser les écoles publiques qui sont sous son autorité directe.

\section{Des équipes de recherche de plus en plus impliquées dans l'organisation même du système éducatif}

C'est donc dans ce cadre que les équipes universitaires impliquées dans la recherche en éducation sont amenées à se développer. Comme le montrent Aubert-Lotarski, Demeuse, Derobertmasure et Friant (2007), ces équipes connaissent, à travers le financement de la recherche et l'intérêt plus ou moins appuyé des responsables du système éducatifs, différentes époques. $\mathrm{Si}$, dans un premier temps, entre le début des années 1960 et la fin du vingtième siècle, se sont surtout les chercheurs eux-mêmes qui influent sur le 
choix des études internationales auxquelles ils souhaitent participer, compte-tenu des moyens budgétaires disponibles et accordés par l'autorité publique. Avec l'arrivée de PISA, le Programme international pour le suivi des acquis des élèves mis en place par l'OCDE, les autorités publiques vont à la fois s'intéresser davantage aux résultats et aux choix des études, privilégiant la participation à cette initiative de l'OCDE. Les études qui avaient jusque là assez peu (voire pas) d'impact sur le système éducatif commencent à susciter l'intérêt des autorités publiques et mettent surtout en évidence un système éducatif très peu régulé et, ce faisant, à la fois fort peu efficace et très inégalitaire.

La double liberté constitutionnelle de 1831 a ainsi principalement conduit à un abandon du système à une régulation de marché, même si les tenants du libre choix s'y réfèrent peu et, le plus souvent, s'en défendent. La justification est davantage celle d'une « mission» dont chacun se sentirait investi, ce terme pouvant, comme dans le cadre de la " Mission de l'école chrétienne » être pris au pied de la lettre si on se réfère au texte qu'édite pour la première fois le Secrétariat général de l'enseignement catholique en 1995 (SEGEC, 2007). En même temps que cette affirmation d'une spécificité - l'enseignement officiel, de son côté, adopte un discours plus construit, un logo...-, les travaux des chercheurs mettent en évidence les effets délétères de cette absence de régulation sur l'efficacité et l'équité du système dans son ensemble (Delvaux et Joseph, 2006 ; Demeuse et Baye, 2007, 2008 ; Demeuse et Friant, 2010, Demeuse, Crahay et Monseur, 2001 ; Dupriez et Dumay, 2011). A côté de la rhétorique qui veut préciser les fondements des choix pédagogiques et éducatifs des réseaux d'enseignement, et les projets de chaque établissement pour justifier le principe de choix, on observe aussi, dans le même texte législatif (Décret "missions », 1997) qui impose la rédaction de ces argumentaires, la volonté d'affirmer des valeurs communes (les quatre missions de l'ensemble du système éducatif, au delà des spécificités des réseaux et des écoles) et de mettre en place des mécanismes régulateurs.

Pour comprendre cette évolution, il faut se reporter quelques années auparavant, comme le précise Lafontaine (2001) :

«Au début des années 90, le Rapport sur l'état du système éducatif rédigé par des experts de l'Ocdé (Ocdé, 1991) met en avant deux caractéristiques inquiétantes du système éducatif en Communauté française de Belgique : l'importance des taux d'échec et la disparité de réussite en fonction de l'établissement fréquenté.

Ce rapport fait grand bruit à l'époque et serait notamment à l'origine de la mise en place, en 1994-95, du dispositif d'évaluation externe. Par ailleurs, l'instauration de celui-ci ne peut se penser qu'en articulation étroite avec une autre initiative d'importance : la définition et mise en place des "socles de compétences" concernant tous les élèves. Des socles de compétences provisoires, élaborés en commun par des représentants des différents réseaux et composantes du système éducatif, voient le jour en 1994. La version définitive de ces socles sera arrêtée en 1999, en même temps que des documents définissant les compétences terminales (fin de l'enseignement secondaire) pour l'ensemble des disciplines concernées.

Il s'agit là d'un événement sans précédent dans l'histoire du système éducatif de la Belgique : pour la première fois, la Communauté française de Belgique se donne un ensemble de références communes, transcendant les programmes particuliers, et il devient donc pleinement légitime, et plus aisé, sur le plan pratique, de mettre en place un dispositif destiné à vérifier dans quelle mesure les élèves atteignent les niveaux de compétences recommandés au moment attendu.

L'initiative de la mise en place d'un dispositif d'évaluation externe revient à un haut responsable (le secrétaire général Jean Magy) de l'Administration générale de l'Enseignement et de la Recherche scientifique du Ministère de la Communauté française de Belgique (Magy, 1998), fortement soutenu dans son initiative par une partie de l'inspection primaire. Même si l'opération a le soutien du Ministère de l'Education de l'époque, elle ne fait cependant l'objet d'aucune disposition législative. 
Par la suite, une cellule responsable de ces évaluations est instaurée au sein de l'administration (Service général, des affaires générales, de la Recherche en Education et du Pilotage interréseaux). Il ne s'agit toutefois pas, loin s'en faut, d'une "direction » à la française (comme celle de l'ancienne DEP ou de l'actuelle DPD) chargée de réaliser les évaluations externes. La responsabilité de cette cellule est d'abord d'ordre administratif : elle définit le calendrier, signe des conventions avec des organismes de recherche extérieurs, coordonne les activités des différents partenaires, apporte un soutien logistique aux évaluations... »

Lafontaine, chercheuse à l'université de Liège et cheville ouvrière de ces évaluations externes non certificatives depuis leur mise en place, poursuit en précisant le caractère original de la démarche, impliquant à la fois une administration qui ne peut s'appuyer sur un cadre législatif spécifique, des équipes de recherche qui reçoivent une commande d'appui technique, des équipes éducatives et l'inspection. Les décisions relatives à ces évaluations s'élaborent sur la base de consensus provisoires. Une fois la définition des compétences à évaluer identifiées, les chercheurs occupent alors un rôle "technique " prépondérant dans le choix des outils d'évaluation, leur préparation, leur mise à l'essai, la sélection de l'échantillon, le recueil et le traitement de données, la présentation des résultats... Les évaluations externes, produites par le système éducatif belge francophone viennent également compléter les informations qui sont fournies par les études internationales, comme le montrent très bien Lafontaine et Blondin (2004).

\section{Une recherche qui se positionne entre prospective et mise en œuvre de politiques concrètes et dépasse ainsi la « simple » évaluation du système}

A côté du travail lié à la gestion des évaluation externes et de PISA - en Communauté française de Belgique, c'est un service universitaire qui a en charge la mise en œuvre de cette évaluation internationale et non l'administration, comme en France, par exemple -, les services universitaires d'enseignement et de recherche, principalement dans les universités publiques, mènent aussi, pour le compte du Gouvernement, dans le cadre de subventions ou de marchés publics, toute une série de travaux, soit de nature exploratoire, soit destinés à permettre la mise en œuvre de politiques spécifiques.

Dans le premier ensemble, on peut citer les travaux de prospectives qui ont été menés par deux équipes interuniversitaires en vue de créer des bassins scolaires et de réguler le financement des établissements de l'enseignement obligatoire en de leur population. Dans le premier cas, cette étude (Delvaux et al. 2005) n'a pas conduit aux modifications en profondeur auxquels certains auteurs pouvaient espérer, même si aujourd'hui on peut trouver quelques traces de ces travaux académiques dans l'organisation du système. Dans le second cas, l'étude interuniversitaire (Demeuse et al. 2007) a permis de revoir une partie de la législation dans le domaine des politiques d'éducation prioritaire, à travers l'encadrement différencié des établissements scolaires qui accueillent des proportions plus importantes d'élèves issus de populations jugées socio-économiquement défavorisées (Demeuse, Demierbe et Friant, 2010; Demeuse, Derobertmasure et Friant, 2010).

Si certaines recherches adoptent une démarche prospective, comme dans les deux cas précédents, certains travaux académiques sont aussi destinés à permettre la mise en œuvre concrète de politiques éducatives. C'est le cas de recherches financées dans le contexte déjà évoqué de la mise en place d'un encadrement différencié des établissements scolaires compte tenu de la population qui les fréquente. Dans ce cadre, et c'est explicitement prévu par la loi (Communauté française de Belgique, 2009, article 3),

«Tous les cinq ans, avant le 30 novembre, et pour la première fois au plus tard le 15 juin 2010, une étude interuniversitaire établit pour la Communauté française l'indice socioéconomique de chaque secteur statistique, c'est-à-dire de chaque subdivision territoriale la plus petite déterminée par la Direction générale Statistique et Information économique du Service public fédéral Economie, Petites et Moyennes Entreprises, Classes moyennes et Energie, au moyen d'une formule de calcul prenant en compte les dernières données statistiques disponibles pour 
les critères suivants : $1^{\circ}$ Revenu par habitant; $2^{\circ}$ Niveau des diplômes; $3^{\circ}$ Taux de chômage, taux d'activité et taux de bénéficiaires du revenu mensuel minimum moyen garanti; $4^{\circ}$ Activités professionnelles; $5^{\circ}$ Confort des logements. "

Cet indice ${ }^{1}$, mis au point par une équipe interuniversitaire est ensuite approuvé par le Gouvernement et utilisé pour affecter des moyens différents aux établissements scolaires. Jusqu'ici - la procédure étant identique depuis 1998 dans le cadre d'un décret précédent - le Gouvernement n'a jamais modifié les résultats produits par l'équipe comportant des chercheurs de trois à quatre universités différentes, publiques et privées. Au-delà de cet objectif précis, le dernier rapport de l'équipe en charge de ce travail (Demeuse et al. 2010) identifie par ailleurs au moins 14 autres usages de cet indice dans le domaine de l'enseignement.

En dehors des évaluations externes où les équipes universitaires jouent un rôle d'appui technique, les travaux décrits jusqu'ici impliquent peu les commanditaires et peu d'interactions sont prévues avec eux durant l'étude, ce qui assure une indépendance recherchée par l'autorité publique de manière à garantir le caractère scientifique des données sur lesquelles elle souhaite s'appuyer.

Dans la suite de ce chapitre, pour montrer l'évolution des interactions, nous aborderons deux exemples assez différents. Le premier concerne la mise au point d'un système d'outils de pilotage et d'aide à la décision, destiné au réseau d'enseignement directement organisé par la Communauté française. Le second exemple que nous présenterons implique le même réseau et notre équipe de recherche, mais concerne cette fois un travail d'analyse des programmes de sciences de l'enseignement secondaire supérieur.

\section{Une recherche-action visant à développer des outils de pilotage du réseau organisé par la Communauté française}

\section{Une position épistémologique parfois complexe et un financement qui l'est tout autant}

Comme nous l'avons vu, le système éducatif belge francophone est assez complexe et son pilotage d'ensemble est délicat. A ce niveau d'organisation, il existe un cadre légal (Communauté française de Belgique, 2002) et une commission de pilotage, dans laquelle siègent les différents acteurs du système, $\mathrm{y}$ compris des chercheurs désignés par le Gouvernement et issus des différentes universités et hautes écoles, qui permettent d'avancer dans cette direction.

Chaque réseau d'enseignement garde cependant une très large autonomie et, selon sa nature, assure la gestion ou la coordination des établissements qu'il regroupe. Au sein du réseau organisé par la Communauté française (nous l'appellerons « le réseau » dans la suite du texte), par opposition aux autres réseaux qui fédèrent des pouvoirs organisateurs indépendants, il existe une ligne hiérarchique qui relie chaque école au ministre compétent à travers différents services et directions de l'administration centrale. Un service général - le service général de l'Enseignement organisé par la Communauté française - est, en particulier, chargé de gérer le réseau. C'est à la demande de celui-ci, dans le cadre d'une subvention de recherche accordée à notre équipe et à une équipe de l'université de Liège, que nous développons un ensemble d'outils de pilotage et d'aide à la décision dont il va être question.

L'objectif principal de cette recherche-action est de doter le réseau d'outils lui permettant, au-delà des outils communs à tous les réseaux, d'avoir une vision claire de son fonctionnement propre et de prendre des décisions adaptées.

Avant d'aller plus loin, il convient sans doute de justifier la terminologie qui est adoptée. La rechercheaction, comme le spécifie le trait d'union reliant les deux termes, nécessite la complémentarité entre la

\footnotetext{
${ }^{1}$ Une présentation du système de calcul de l'indice peut être trouvée dans Demeuse et Monseur (1999).
} 
recherche et l'action (Brabant, 2006, p. 12). La recherche-action peut se décliner de différentes manières : (1) sous la forme d'une recherche sur l'action, de nature explicative, (2) d'une recherche pour l'action, de nature appliquée et (3) d'une recherche par l'action, de nature impliquée (Goyette et Lessard-Hébert, 1987). L'idée principale d'une recherche-action est la production de savoirs dans et par l'action de groupes sociaux. A partir d'une situation donnée (qu'elle soit pensée à partir d'un besoin local ou à une réflexion davantage théorique), on induit un changement et on étudie les conditions et les résultats de cette expérimentation, ce qui permet, d'une part, d'améliorer le contexte dans lequel ce changement a eu lieu et, d'autre part, de parfaire le savoir existant (Brabant, 2006, p. 13). Dans ce contexte, l'équipe de recherche est partie prenante à la fois dans l'évolution de la situation, mais aussi, ce qui est sans doute plus classique en théorie pour des chercheurs, dans la production de connaissances. Le risque existe cependant de privilégier l'action au détriment du développement des connaissances, comme le souligne Clermont (1986).

De manière symétrique, l'organisation - ici, les cadres du réseau - est impliquée dans l'évolution de sa propre situation, mais aussi dans la production de connaissances que les chercheurs devront sans doute formaliser pour les incorporer aux connaissances "savantes" et communicables en dehors de l'organisation elle-même. Il ne s'agit donc pas de fournir un service ${ }^{2}$, ni même de réaliser un transfert de technologie sur la base d'un cahier des charges initial spécifiant l'ensemble des " délivrables ». C'est bien ce qui fait de cette démarche une approche particulière, impliquant un cadre éthique spécifique, basé sur la confiance (et non un contrat commercial), qui ne saurait être soumise aux mêmes contraintes, par exemple en matière de TVA, puisque, comme en matière d'éducation, la réalisation finale dépend à la fois de tous les partenaires, y compris ceux qu'on pourrait baptiser " bénéficiaires », et de leur implication dans le processus. Le terme «bénéficiaire » est cependant peu adapté au contexte de notre recherche-action : les bénéficiaires finaux d'une amélioration du système éducatif n'en sont pas les cadres, mais... les élèves (qui, dans le cas présent, ne sont pas directement impliqués dans le processus) et à travers eux, la société.

Par ailleurs, s'il convenait d'estimer la valeur ajoutée du processus, il faudrait aussi y inclure les productions scientifiques qui en résultent à court, moyen et long termes. Ici aussi, cette production, sauf à considérer les brevets qui pourraient être déposés et leur valorisation ultérieure, n'est pas soumise à une évaluation marchande et ne relève donc pas du régime ordinaire de la TVA (hors champ). Cette discussion n'est pas anodine en regard des difficultés que nous rencontrons actuellement en matière de statut de la recherche et de la recherche commanditée par des organisations publiques, en particulier, y compris au sein de certaines universités, considérées à présent comme des assujettis mixtes.

De manière plus concrète sans doute, le projet qui est dans sa troisième année, a permis, en interaction avec les responsables du réseau et d'autres directions de l'administration, de développer une application informatique qui permet d'interroger, en utilisant un gestionnaire de base de données, un entrepôt de données, constitué essentiellement de données déjà collectées par l'administration à différents sujets, sans devoir réinterroger les directions des écoles ou d'autres acteurs de terrain. Il s'agit là de la partie la plus visible.

Cette application intègre trois outils interconnectés qui ont des objectifs distincts (Hourez et al. à paraître). L'objectif d'un premier outil est de permettre une modélisation dynamique de la distribution de la population d'élèves et des flux entre établissements scolaires, permettant à la fois de mieux comprendre les mécanismes à l'œuvre et de tester l'évolution du système sous différents scénarios (Hourez et al. 2011; Friant et al. 2011). Le deuxième outil offre la possibilité d'effectuer des recherches d'établissements, sur la base de différents critères comme par exemple, les filières et options de cours réellement proposées par les établissements, de l'existence de moyens de transports en commun entre le domicile de l'élève et l'établissement, des restrictions d'orientation posées par le conseil de classe de l'élève, etc. Le dernier outil, s'inspirant des Indicateurs de l'enseignement (ETNIC/Fédération Wallonie-

\footnotetext{
${ }^{2}$ Manoukian (2001) préfère parler «d'issue » plutôt que de « résultat» dans le cadre d'une recherche-action. Pour notre part, nous utilisons le terme « réalisation » pour indiquer ce qui résulte finalement du processus.
} 
Bruxelles, 2011), présente, au travers d'une interface relativement simple d'utilisation, des indicateurs décrivant les caractéristiques propres à l'enseignement au sein du réseau, tels que les caractéristiques du public scolarisé, les parcours typiques des élèves dans le réseau, la situation du personnel ou des infrastructures... Cet ensemble est orienté vers la régulation que peuvent exercer effectivement les acteurs sur les différents niveaux, aussi bien de manière centrale que dans les écoles elles-mêmes.

La réalisation de ce travail, par son caractère interactif, a aussi permis de questionner certains circuits de communications et certaines manières de faire... et de les améliorer. Ainsi, par exemple, il conduit à repréciser certaines collectes de données (c'est le cas, par exemple, dans le domaine de évaluations externes) et à étudier la manière de transmettre les informations de façon à les rendre réellement utilisables et utilisées, à la fois par les cadres du réseau, mais également par les écoles. Il a aussi permis diverses publications en rapport avec les préoccupations de notre équipe de recherche dans le domaine de la régulation des systèmes pour favoriser à la fois l'efficacité et l'équité de celui-ci (Demeuse et al. 2005 ; Nicaise, Desmedt et Demeuse, 2009).

\section{Quelques conditions permettant de clarifier le statut de la recherche et des chercheurs}

Cette démarche repose sur quelques conditions que l'équipe de recherche doit s'efforcer de maintenir. Pour commencer, et c'est sans doute le plus important, le point de départ doit s'ancrer dans des questionnements scientifiques propres aux sciences de l'éducation tels que comprendre comment fonctionne le système éducatif, comment une situation particulière réagit sous l'effet d'une action ou identifier comment améliorer une action. Il ne suffit donc pas de répondre à une demande.

Il faut par ailleurs avoir une certaine légitimité dans le domaine, ce qui distingue les services de recherche des universités des officines polyvalentes qui proposent leurs services de manière très large à des organisations pourtant très différentes. On ne peut pas se dispenser d'une revue des connaissances théoriques, pratiques (expériences) et technologiques (outils) actuelles. Celle-ci doit être assez large pour permettre de rencontrer de manière ouverte les problèmes qui ne vont pas manquer de se présenter et qui n'auraient pas été anticipés au départ.

La mise en place d'un processus de modification des connaissances actuelles (modélisation, et développement d'outils et de démarches) doit être encadrée par une approche scientifique de récolte de données, d'analyses, d'évaluations et d'adaptations éventuelles du processus. La diffusion de résultats de recherche, qui incluent les connaissances scientifiques préalables à la recherche et les acquis scientifiques issus de la recherche, fait intégralement partie du processus et doit avoir été anticipée pour permettre sa réalisation dans des conditions acceptables. La communication des nouveaux acquis scientifiques et des résultats à la communauté scientifique, tant en cours de recherche qu'au terme de celle-ci, pour partager les connaissances et outils développés, valider les pratiques et les démarches ou ouvrir un débat éventuellement contradictoire, est indispensable à l'entretien de ce type de démarche. C'est d'ailleurs à la démarche, et non à l'obtention de résultats souhaités ou prévus que doit être conditionné le financement de ce type de recherches, ce qui les distinguent bien de la production de services.

\section{Analyser les programmes dans l'enseignement secondaire supérieur pour les rendre plus cohérents ou revoir la structure de l'enseignement en profondeur?}

\section{Une recherche en apparence mieux cadrée...}

Le type de démarche qui vient d'être décrit peut être appliqué dans d'autres domaines de l'évaluation. Nous avons ainsi pu travailler dans le domaine de l'analyse des programmes de sciences (physique, chimie et biologie) dans l'enseignement secondaire du réseau organisé par la Communauté française. 
Si la démarche pouvait sembler plus cadrée, construite autour des trois niveaux du curriculum (prescrit, implanté et réalisé), selon une méthode assez classique (Demeuse et Strauven, 2006), l'état des documents " programmes" (niveau prescrit) et l'actualité (révision des référentiels communs à tous les réseaux) a conduit progressivement à adopter une approche plus ouverte. Bien qu'au départ donc, les chercheurs aient imaginé de travailler à l'amélioration des documents programmes de manière à en faciliter la compréhension par les enseignants, il s'est vite avéré indispensable de reconsidérer le problème de manière beaucoup plus globale. Au-delà de la qualité des documents eux-mêmes, c'est la cohérence de tout l'édifice curriculaire qui devait être améliorée.

L'analyse des curricula et des politiques curriculaires constitue - c'est relativement nouveau dans le monde francophone ${ }^{3}$ - un domaine qui permet de s'intéresser de manière globale à un système éducatif, depuis la définition de ce qu'il faut apprendre, jusqu'à sa maîtrise (ou non) par les élèves.

\section{... mais qui se heurte très vite à la complexité du système}

Avant même de se rendre dans les classes, il est possible d'analyser la manière dont le système planifie les apprentissages (Stufflebeam, 2002) et il est fort probable, en regard de l'analyse que nous avons menée, que tous les élèves ne pourront atteindre les objectifs visés du fait même de cette structure. Malheureusement, en effet, pour reprendre les termes de Jonnaert (2011, p. 141), «tout curriculum [...], défini par des systèmes rationnels, [...] évolue dans l'irrationalité d'une société donnée ».

L'organisation de notre enseignement secondaire, en particulier, n'a rien de simple, nous l'avons déjà souligné. Elle a subi de nombreuses réformes qui l'ont rendue de moins en moins lisible, tout en proposant, sur papier du moins, un modèle d'intégration qui permet de passer d'une forme ou d'une filière à l'autre, voire d'accéder à l'enseignement supérieur universitaire après une formation professionnelle, alors même que des finalités différentes sont assignées à ces formes et à ces filières. Tout élève est donc, théoriquement, libre de choisir et de changer de forme et de filière, dans les limites fixées par les conseils de classe (Demeuse \& Lafontaine, 2005). A cela s'ajoute encore la possibilité bien réelle de changer d'école et donc, de pouvoir organisateur et de réseau...

Ces choix génèrent autant de parcours, qui croiseront autant de programmes d'études et conduiront à poursuivre, selon la filière ou la forme d'enseignement fréquentée, autant de finalités différentes ${ }^{4}$. La couverture par les programmes s'étendant de la $3^{\mathrm{e}}$ à la $6^{\mathrm{e}}$ année de l'enseignement secondaire, selon l'option et la filière choisie (sciences générales, sciences de base, éducation scientifique, ou sciences dans les filières techniques de qualification et professionnelles) peut être extrêmement différente, comme l'a montré notre étude (Soetewey, Duroisin et Demeuse, 2011). Ces données mettent notamment en évidence, dans le réseau organisé par la Communauté française ${ }^{5}$, la réduction de la couverture des programmes en termes de connaissances (savoirs) lorsqu'on passe de l'enseignement de transition (général) vers l'enseignement de qualification (professionnel).

Par surcroit, les balises générales fournies pour l'ensemble du système (socles de compétences et compétences terminales) laissent une très large liberté, ne serait-ce que dans le phasage et l'ordonnancement des contenus. Ainsi, entre la fin de la deuxième année et la fin de la sixième année de l'enseignement secondaire, les pouvoirs organisateurs ou leurs organes de représentation seront-ils libres

\footnotetext{
${ }^{3}$ Voir à ce sujet le numéro qu'y consacre la Revue internationale d’Education. Sèvres, d'avril 2011 (n56) et les ouvrages de Audigier \& Tutiaux-Guillon (2008), Audigier, Crahay \& Dolz (2006), Demeuse \& Stauven (2006), Depover \& Noel (2005), Ettayebi, Jonnaert \& Opertti (2008), Forquin (2008) ou Mangez (2010).

${ }^{4}$ Pour les sciences, dans le deuxième et le troisième degrés de l'enseignement secondaire, les Compétences terminales et savoirs requis en sciences générales, les Compétences terminales et savoirs requis en sciences de base ou les Compétences terminales et savoirs communs.

${ }^{5}$ Ce type de constat n'est pas propre à ce réseau. Hirtt (2005), comparant l'enseignement de la physique dans le réseau organisé par la Communauté française et dans le réseau libre catholique, analyse de manière extrêmement sévère les programmes de ce dernier et parle même de "pédagogie de l'incompétence", face au peu de place qui y est laissée aux savoirs (http://www.skolo.org/spip.php?article275). Mangez (2004) en donne une image certes moins extrême, mais convergente.
} 
de proposer des programmes qui disposent de ce qui sera vu en troisième, en quatrième ou ailleurs, sans concertation obligatoire avec les autres réseaux qui proposeront, eux-aussi, leur propre agencement, localement rationnel. On imagine immédiatement que ce qui est localement rationnel a très peu de chance de l'être globalement... si rien n'oblige à se concerter et si la «logique de la matière » ne l'impose pas. Or, le passage d'une approche par les contenus, beaucoup plus facile à programmer, abandonnée au profit d'une approche par les compétences, très difficilement programmable, n'est pas de nature à simplifier cette organisation. L'absence de manuels officiels (il existe bien des manuels agréés, mais ils ne sont pas obligatoires et leur structure n'est évidemment ni imposée ni commune puisqu'aucun texte ne pourrait soutenir une telle imposition) laisse entrevoir un système plus compliqué que complexe, pour utiliser des mots convenables...

On l'aura compris, la solution qui permet le mieux de tirer parti d'une telle organisation, si on veut réellement atteindre l'un des ensembles de finalités décrites pour l'enseignement secondaire, consiste à éviter les bifurcations et les changements. Il faudrait pouvoir maintenir le cap et profiter des rationalités locales à travers un enchaînement de programmes cohérents, et donc éviter de changer de filière, de forme, d'option et surtout d'école et de réseau... en un mot, de ne pas profiter de cette immense liberté en trompe l'œil. Cette conclusion, assez simple à tirer si on se penche sur notre système, certains l'ont bien comprise... Il s'agit (des parents) des élèves qui poursuivent sans encombre un enseignement général dans l'école où ils ont pris leurs quartiers dès la première année. Pour les autres, la situation sera sans doute moins favorable et pour ceux qui cumuleront (ré)orientation, redoublement, changement(s) d'école... la situation scolaire moins favorable qu'ils connaissent déjà du fait de leur moindre compétence académique sera encore amplifiée par les incohérences globales d'un système construit pour permettre la «libre circulation » et non l'optimisation des apprentissages de tous.

A la lecture de ces quelques lignes, le lecteur aura sans doute perçu que cette «libre circulation » ne profite pas de la même manière à tous les élèves. Il est d'ailleurs piquant de constater que ce sont ceux qui « circulent le moins » qui profitent le mieux du système... pour apprendre ou, du moins, faire reconnaître leurs apprentissages. Notre système n'en est pas à un paradoxe près... mais une analyse minutieuse des programmes, c'est-à-dire de ce qui est prescrit, avant même de se rendre dans les classes ou d'évaluer les apprentissages réels des élèves à travers évaluations externes et études internationales du type de PISA, permet de conclure que notre système a bien peu de chances d'être efficace et/ou équitable... comme le confirme l'ensemble des études qui lui sont consacrées. Pour palier le problème, cela ne suppose pas de placer les enseignants dans un carcan absolu, mais conduit à fournir des balises organisationnelles beaucoup plus précises qu'aujourd'hui. Ces balises doivent être articulées à travers tout le système et, faute d'une intelligence spontanée et collective de tous les acteurs visant l'intérêt des enfants et non la préservation de parts de marché, la Communauté française doit mieux décrire ce qui est attendu, d'une part, d'une formation scientifique pour tous, quel que soit le parcours suivi (y compris à quel moment il convient d'enseigner tel ou tel concept) et, d'autre part, d'une formation spécialisée. Cela repose la question de la longueur et du contenu du tronc commun, et du contrôle effectif du niveau des études, pourtant prescrit dans la loi dite du Pacte scolaire, dès 1959.

Un autre enseignement important de ce type d'analyse concerne les enseignants. S'il existe sans doute des enseignants plus efficaces que d'autres, une telle structure va, plus que probablement, ne pas leur faciliter la vie, du moins à ceux qui n'enseignent pas dans les écoles qui peuvent se permettre d'écrémer en réorientant une partie de leurs effectifs vers les autres établissements. Cette organisation a en effet toute les chances, en dehors des filières les plus sélectives, de produire à la fois un abaissement général du niveau moyen des élèves et une hétérogénéisation maximale des compétences individuelles de ceux-ci, quelles que soient les qualités pédagogiques des enseignants. Encore une fois, ce qui peut se déduire aisément de l'analyse du curriculum prescrit s'observe sur le terrain au travers des différentes enquêtes et études empiriques relatives aux performances des élèves, c'est-à-dire du curriculum réalisé. 


\section{Conclusions}

Evaluer est une composante essentielle de tout processus régulé. Le système éducatif belge francophone a été, pendant très longtemps et reste encore, assez peu régulé. Les évaluations des acquis des élèves, parmi lesquelles on retrouve les évaluations internationales auxquelles les chercheurs en sciences de l'éducation contribuèrent fortement, faute de volonté et compétences au sein du système avant 1990, ont contribué, en retour, à accroître les compétences des équipes universitaires dans ce domaine. Ces compétences ont alors pu être mobilisées lorsque certains décideurs sont devenus plus sensibles à l'intérêt d'une régulation plus poussée d'un système qui s'orientait résolument vers une situation de plus en plus régie par le marché et les rapports de forces.

Au travers des deux exemples contrastés qui ont été présentés, nous avons voulu souligner, d'une part, la possibilité de développer une démarche participative de type recherche-action visant à la fois l'amélioration d'un processus et une meilleure connaissance d'un système complexe et, d'autre part, l'importance de la prise en compte d'autres éléments que les résultats à des tests standardisés, même si ceux-ci sont importants, pour piloter un système. On ne peut, en effet, faire l'économie de l'analyse des curricula, même si celle-ci est complexe et pose des problèmes pas toujours anticipés. Nous avons ainsi constaté rapidement qu'il ne suffirait pas de faire du « cosmétique » en toilettant des programmes pour les rendre plus lisibles, mais qu'il faudrait accepter de considérer le système dans son ensemble si on souhaitait vraiment l'améliorer. Du point de vue de l'amélioration, le premier exemple qui a été analysé est sans doute plus satisfaisant en termes d'avancement. Le second n'a encore produit, pour sa part, aucun résultat tangible, si ce n'est la plus grande sensibilisation des décideurs à la problématique de la construction des programmes et à l'agencement des parcours. Il ne suffit donc pas d'évaluer pour progresser... mais il est impossible de progresser sans évaluer. Reste qu'il n'est pas non plus possible de progresser si l'on ne sait pas vers où on souhaite aller... ce qui ne relève pas de la compétence des chercheurs, mais bien du politique.

\section{Bibliograhie}

Aubert-Lotarski, A., Demeuse, M., Derobertmasure, A., \& Friant, N. (2007). Conseiller le politique: des évaluations commanditées à la prospective en éducation, Les Dossiers des Sciences de l'Education, 18, 121130.

Audigier, F., Tutiaux-Guillon (éds.)(2008). Compétences et contenus. Les curriculums en question. Bruxelles : De Boeck université.

Audigier, F., Crahay, M., Dolz, J. (éds.)(2006). Curriculum, enseignement et pilotage. Bruxelles : De Boeck université.

Brabant, L. (2006). Le concept d'intervention dans les champs disciplinaires des sciences humaines, Cahiers de recherche de la Chaire d'Ethique appliquée, $n^{\circ} 32$.

Gauthier, C. (1986). Les rapports entre la théorie et la pratique et la recherche-action, Revue des sciences de l'éducation, vol. 12, n 3, 331-343.

Communauté française de Belgique (2009). Décret du 30 avril 2009 organisant un encadrement différencié au sein des établissements scolaires de la Communauté française afin d'assurer à chaque élève des chances égales d'émancipation sociale dans un environnement pédagogique de qualité. Paru au Moniteur belge du 9 juillet 2009.

Communauté française de Belgique (2002). Décret du 27 mars 2002 relatif au pilotage du système éducatif de la Communauté française. Paru au Moniteur belge le 17 mai 2002.

Delvaux, B., \& Joseph, M. (2006). Hiérarchie scolaire et compétition entre écoles : le cas d'un espace local belge. Revue française de pédagogie, 156, 19-27.

Delvaux, B., Demeuse, M., Dupriez, V., Fagnant, A., Guisset, C., Lafontaine, D., Marissal, P., Maroy, C. (juillet 2005). Les bassins scolaires: de l'idée au projet. Propositions relatives aux domaines d'intervention, aux instances et aux territoires. Bruxelles : Rapport à la Communauté française de l'étude intitulée «Définition, 
cartographie, rôles et modes de régulation des bassins scolaires ». http://www.enseignement.be/index.php?page=26044\&id fiche $=4880 \&$ dummy $=25120$ consulté le 4 mars 2011.

Demeuse, M. (2010). L'école peut-elle être juste et efficace? Politique, Hors-série n¹5, 6-18.

Demeuse, M., \& Baye, A. (2007). Measuring and Comparing the Equity of Education Systems in Europe. In N.C. Soguel \& P. Jaccard (eds), Governance and Performance of Education Systems. Dordrecht (NL): Springer. (pp. 85-106)

Demeuse, M., \& Baye, A. (2008). Indicateurs d'équité éducative. Une analyse de la ségrégation académique et sociale dans les pays européens. Revue française de pédagogie, 165, 91-103.

Demeuse, M., \& Friant, N. (2010). School segregation in the French Community of Belgium. In J. Bakker, E. Denessen, D. Peters, \& G. Walraven (Éds.), International perspectives on countering school segregation. Apeldoorn: Garant. (pp. 173-192).

Demeuse, M., Lafontaine, D. (2005). L'orientation scolaire en Communauté française de Belgique. Revue internationale d'éducation Sèvres, 38, 35-51.

Demeuse, M., Monseur, C. (1999). Analyse critique des indicateurs déterminant l'attribution des moyens destinés à la politique de discrimination positive en Communauté française de Belgique. Mesure et Evaluation en Education, 22(2-3), 97-127.

Demeuse, M., Strauven, C. (2006). Développer un curriculum d'enseignement ou de formation. Des options politiques au pilotage. Bruxelles : De Boeck université, Coll. Perspectives en éducation et formation

Demeuse, M., Crahay, M., \& Monseur, C. (2001). «Efficiency and Equity ». In W. Hutmacher, D. Cochrane, N. Bottani. In Pursuit of Equity in Education. Using international indicators to compare equity policies. Doordrecht: Kluwer (pp. 65-91).

Demeuse, M., Demierbe, C., Friant, N. (2010). Des ZEP à l'encadrement différencié, vingt ans de politiques compensatoires en Communauté française de Belgique. InDirect, les Clés de la gestion scolaire, 18, 65-78.

Demeuse, M., Derobertmasure, A., \& Friant, N. (2010). Differentiated financing of schools in French-speaking Belgium: prospectives for regulating a school quasi-market. Educational Research and Evaluation: An International Journal on Theory and Practice, Volume 16, Issue 2, $197-216$.

Demeuse, M., Baye, A., Straeten, M.H., Nicaise, J., Matoul, A. (éds). (2005). Vers une école juste et efficace. 26 contributions sur les systèmes d'enseignement et de formation. Bruxelles: De Boeck Université, collection "Economie, Société, Région".

Demeuse, M., Dandoy, A., Delvaux, B., Franquet, A., Friant, N., Marissal, P., Monseur, C., \& Quittre, V. (2010). Actualisation de l'indice socio-économique des secteurs statistiques en application de l'article 3 du décret du 30 avril 2009 organisant un encadrement différencié au sein des établissements scolaires de la Communauté française. Rapport de l'équipe interuniversitaire à Madame la Ministre Marie-Dominique Simonet en charge de l'enseignement obligatoire. Rapport non publié.

Demeuse, M., Derobertmasure, A., Friant, N., Herremans, T., Monseur, C., Uyttendaele, S., \& Verdale, N. (2007). Etude exploratoire sur la mise en cuvre de nouvelles mesures visant à lutter contre les phénomènes de ségrégation scolaire et d'inéquité au sein du système éducatif de la Communauté française de Belgique. Rapport réalisé à la demande du Gouvernement de la Communauté française de Belgique. http://www.enseignement.be/index.php?page=26044\&id fiche=5176\&dummy=25981 consulté le 4 mars 2012.

Depover, C., Noel, B. (2005).Le curriculum et ses logiques : une approche contextualisée pour analyser les réformes et les politiques éducatives. Paris : L'Harmattan.

Dupriez, V., \& Dumay, X. (2011). Les quasi-marchés scolaires: au bénéfice de qui ? Revue française de pédagogie, 176, 83-100.

ETNIC/Fédération Wallonie-Bruxelles (2011). Les indicateurs de l'enseignement 2011. Bruxelles : ETNIC/Fédération Wallonie-Bruxelles. 
Ettayebi, M., Jonnaert, P., Opertti, R. (éds.)(2008). Logique de compétences et développement curriculaire. Débats, prespectives et alternative pour les systèmes éducatifs. Aris : L'Harmattan.

Friant, N., Soetewey, S., Demeuse, M., Hourez, J., Wijsen J. (2011) Le système éducatif: un système complexe que l'on peut modéliser. Elément : le Magazine de l'Université de Mons, 5, 43-45.

Forquin, J.C. (2008). Sociologie du curriculum. Rennes : Presses universitaires de Rennes.

Jonnaert, P. (2011). Curriculum, entre modèle rationnel et irrationalité des sociétés. Revue internationale d'Education. Sèvres, $n^{\circ} 56,135-145$.

Hourez, J., Friant, N., Soetewey, S., \& Demeuse M. (2011). Le comportement individuel des élèves, une clé pour modéliser la dynamique du système éducatif. In XVIII èmes Journées d'études sur les données longitudinales dans l'analyse du marché du travail: Les nouvelles ségrégations scolaires et professionnelles, Relief $n^{\circ} 34$, 131-145.

Hourez, J., Soetewey, S., Crépin, F., \& Malaise, S. (à paraître). Mise en place d'un outil de pilotage effectif pour exploiter les évaluations externes non certificatives à différents niveaux. Actes du $24^{e}$ colloque international de l'ADMEE-Europe.

Goyette, G., \& Lessard-Hébert, M. (1987). La recherche-action: ses fonctions, ses fondements et son instrumentation. Québec : Presses de l'Université de Québec.

Lafontaine, D. (2001). Le dispositif d'évaluation externe en Communauté française de Belgique [Recherche en éducation no80/99]. Le Point sur la Recherche en Education, $n^{\circ} 19$ (mars 2001). www.enseignement.be/download.php?do id=1855\&do check= consulté le 25 février 2012.

Lafontaine, D. (2009). Bien avant PISA, enquêtes internationales et évaluations externes en Communauté française: approches différentes, constats largement convergents. www.enseignement.be/download.php?do id=5295\&do check= consulté le 25 février 2012.

Lafontaine, D., \& Blondin, C. (2004). Regards sur les acquis des élèves en Communauté française de Belgique. Apports des enquêtes de l'IEA, de Pisa et des évaluations externes. Bruxelles : De Boeck, Pédagogies en développement.

Mangez, E. (2004), « La production des programmes de cours par les agents intermédiaires : transfert de savoirs et relations de pouvoir », Revue Française de Pédagogie, $n^{\circ}$ 146, $65-77$.

Mangez, E. (2010). Réformer les contenus d'enseignement: une sociologie du curriculum. Paris : Presses universitaires de France.

Manoukian, F. (2001). Prémisses et issues de la recherche-action, Revue internationale de Psychosociologie, 16-17 (Vol. VII), 235-249.

Nicaise, I., Desmedt, E., Demeuse, M. (éds) (2009). Une école réellement juste pour tous ! Seize pistes de réforme et d'action. Waterloo : Plantyn.

SEGEC (2007). Mission de l'Ecole chrétienne. Bruxelles: Secrétariat général de l'enseignement catholique. http://enseignement.catholique.be/segec/index.php?id=803 consulté le 25 février 2012.

Soetewey, S., Duroisin, N., Demeuse, M. (2011). Le curriculum oublié. Analyse comparée des programmes de sciences en Belgique francophone. Revue internationale d'Education. Sèvres, 56, 123-134.

Stufflebeam, D. L. (2002). Foundational models for the 21st century program evaluation. In D. L. Stufflebeam, G. F. Madaus, \& T. Kellaghan (Eds.), Evaluation Models Viewpoints on educational and human services evaluation (p. 33-83). Netherlands: Springer.

Vandenberghe, V. (1998). L'enseignement en Communauté française de Belgique : un quasi-marché. Reflets et perspectives de la vie économique, 26(1), 65-75. 